\title{
Path tracking with minimum time of vehicle handling inverse dynamics based on adaptive gauss pseudospectral method
}

\author{
Yongsheng Liu ${ }^{1}$, Yingjie Liu ${ }^{2}$ \\ School of Mechanical-Electronic and Vehicle Engineering, Weifang University, \\ Weifang, 261061, Shandong, China \\ ${ }^{2}$ Corresponding author \\ E-mail: ${ }^{1}$ liuys@wfu.edu.cn, ${ }^{2}$ foliuyingiie@163.com
}

Received 24 June 2019; received in revised form 17 September 2019; accepted 22 October 2019 DOI https://doi.org/10.21595/jme.2019.20875

Check for updates

Copyright (C) 2019 Yongsheng Liu, et al. This is an open access article distributed under the Creative Commons Attribution License, which permits unrestricted use, distribution, and reproduction in any medium, provided the original work is properly cited.

\begin{abstract}
Vehicle driving safety is the urgent key problem to be solved by an independent automobile development project. And it is also the premise and one of the necessary conditions for active vehicle safety. A new technique for path tracking with minimum time of vehicle handling inverse dynamics is proposed in this paper. Based on a new optimal control method Adaptive Gauss Pseudospectral Method (AGPM), the optimal control for path tracking with minimum time problem was converted into a nonlinear programming problem which met the boundary condition and a series of path constraints including path constraint, state constraint, control constraint. And then the problem was solved using the sequential quadratic programming (SQP). The simulation results showed that the proposed method was not sensitive to the initial value and the optimization efficiency was higher compared with indirect methods and traditional direct methods. When solving the problem of path tracking with minimum time as per this method, boundary constraints and path constraints were well satisfied. The algorithm was not only precise but it could also shorten the evaluation period of vehicle handling stability and could reduce the tremendous cost for real vehicle testing. So the maneuverability of two different vehicles that complete the pylon course slalom test road with minimum time can be evaluated objectively by utilizing the method. The model correctness is verified through a real vehicle test.
\end{abstract}

Keywords: path tracking, AGPM, inverse problem, minimum time.

\section{Introduction}

Driving a vehicle is a process that driver generates vehicle trajectories through detecting and recognizing obstacles on the road and then drives the vehicle along the planned trajectories. The driver maneuvers, and driving tasks are updated timely when the driving environment and the vehicle state are changing over time. But the driver modeling method is always a "bottleneck" problem in the driver-car closed-loop system dynamics. As a result, the inverse vehicle handling dynamics which can solve the driver's input based on known vehicle model and state of the vehicle motion (the vehicle response) is called the "inverse approach" of vehicle handling dynamics. Trajectory tracking control was one of the most advancing topics for the past decade [1-4].

Ever since the invention of automobiles, research in vehicle safety systems has been an ongoing study. Engineers and researchers have been trying to fully understand the dynamic behavior of vehicles as they are subjected to different driving conditions, with different drivers. They want to apply these findings to improve issues such as ride quality and vehicle stability and safety, and to develop innovative designs that will improve vehicle operation $[5,6]$.

The problem of path tracking with minimum time has been studied in the literatures. A brief review is presented in what follows.

Yang et al. [6] presented a theoretical expansion of a new intelligent algorithm called as the extended support vector data description (E-SVDD) for the analysis and control of dynamic groups to realize macroscopic and microscopic behavior prediction in an automotive collision 
avoidance system. However, the algorithm's effectiveness is ignored.

$\mathrm{Fu}$ et al. [7] proposed a path tracking controller for an autonomous vehicle, which aims at pushing it to the driving and handling limit. The limit dynamic performance of the autonomous vehicle was represented by the G-G diagram, which indicated the acceleration capability of the autonomous vehicle. The simulation validation results demonstrated the effectiveness of the proposed controller. However, such important information as robustness data about the algorithm is ignored.

Krid et al. [8] presented a path tracking controller for a fast rover with independent front and rear steering. The controller was based on the dynamic model of a bicycle like vehicle which considered the lateral slippage of the wheels. The prediction model allowed the anticipation of future changes in set points in accordance with the dynamic constraints of the system. Experimental results showed a good control accuracy and appeared to be robust with respect to environmental and robot state changes. But there was a strong dependency on the precision of the model.

Amer et al. [9] presented a development and optimization of a proposed path tracking controller for an autonomous armored vehicle and developed a path tracking control based on an established Stanley controller for autonomous vehicles. The basic controller was modified and applied on a non-linear, 7-degrees-of-freedom armored vehicle model, and consisted of various modules such as handling model, tire model, engine, and transmission model. But the proposed method bears heavier computing burden.

Guo et al. [10] presented a novel coordinated path following system (PFS) and direct yaw-moment control (DYC) of autonomous electric vehicles via hierarchical control technique. Finally, the effectiveness of the proposed control system was validated by the simulation and experimental tests. But there was difficulty in rule-making.

Memon et al. [11] presented a comprehensive architecture of an active collision avoidance system for an autonomous vehicle to deal with potential hazards on a straight road or a curved road. Meanwhile, the robustness about the algorithm is ignored in this process.

Setiawan et al. [12] presented a new type of four-wheel independent steering automatic guided vehicle (4WISAGV) for carrying heavy luggage and proposed a controller for the 4WIS-AGV to track reference trajectories. The results showed that the proposed controller could make the 4WIS-AGV track the trajectory with sharp edges and the circular trajectory very well.

Zou et al. [13] proposed a novel approach to the dynamic modeling and motion control of tracked vehicles undergoing skid-steering on horizontal, hard terrain, under non-holonomic constraints. Validated via a numerical example, the proposed controller was proven to be effective in controlling an unmanned tracked vehicle. However, the external disturbance was studied insufficiently.

$\mathrm{Yu}$ et al. [14] designed a novel controller to improve the path-tracking performance of articulated dump truck (ADT). The designed controller combined with a linear quadratic regulator (LQR) with genetic algorithm (GA) was used to control linear and angular velocities on the midpoint of the front frame. The results of simulation and experiment showed that the LQR-GA controller had a better tracking performance than the existing methods under a low speed of $3 \mathrm{~m} / \mathrm{s}$. However, such important information as robustness data about the algorithm is ignored.

Wang et al. [15] described a control framework to address a typical vehicle-to-vehicle encountering scenario of lane exchanging with two vehicles which traveled on contiguous lanes in the same direction and were maneuvered to exchange lanes of each other. A trajectory generating model for lane-changing maneuver was integrated into the driver-vehicle system. While the roll condition performance of the vehicle should be discussed.

This paper aims to present a method which is based on the optimal control theory for path tracking problem in inverse vehicle handling dynamics. The method is used to calculate the optimal control input such as steering torque for a vehicle tracking the desired path with the minimum time without striking neighboring obstacles or departing the desired path almost. Focusing on the purpose, this paper presents a 4-degrees-of-freedom (DOF) vehicle model firstly. 
And also, we establish a simulation to verify the effectiveness of the proposed method and propose a comparative verification with another traditional method. After verifying the correctness of the numerical simulation by experiment, the conclusions are summarized and the future research directions are suggested.

\section{Model of vehicle path tracking problem}

\subsection{Mathematical model of vehicle path tracking with minimum time problem}

It is assumed that the longitudinal force acting on the front wheels is small, and the influence on the tire cornering characteristics affected by tangential ground force is ignored with tire cornering characteristics within the linear range. The 4-DOF vehicle model is depicted in Fig. $1[16]$.

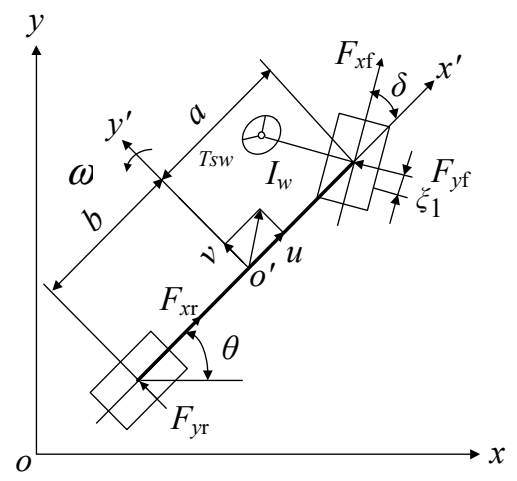

Fig. 1. 4-DOF vehicle model

In the state-space form, the formula is as follows:

$$
\left\{\begin{array}{l}
\dot{u}=v \omega+\frac{F_{x f} \cos \delta-F_{y f} \sin \delta+F_{x r}-F_{f}-F_{w}}{m}, \\
\dot{v}=-u \omega+\frac{F_{y f} \cos \delta-F_{y r} \sin \delta+F_{x f} \sin \delta}{m}, \\
\dot{\omega}=\frac{a F_{y f} \cos \delta-b F_{y r}+a F_{x f} \sin \delta}{I_{z}}, \\
\dot{\delta}=p, \\
\dot{p}=-\frac{k_{1} \xi_{1}}{I_{w} u} v-\frac{k_{1} \xi_{1} a}{I_{w} u} \omega+\frac{\left(k_{1} \xi_{1}-k_{w}\right)}{I_{w}} \delta-\frac{c_{w}}{I_{w}} p+\frac{T_{s w} i}{I_{w}} .
\end{array}\right.
$$

The parameters and corresponding definitions are shown in Table 1.

The lateral forces of the front and rear tires are:

$$
\left\{\begin{array}{l}
F_{y f}=k_{1}\left(\frac{v+a \omega}{u}-\delta\right) \sqrt{1-\left(\frac{F_{x f}}{\mu F_{z f}}\right)^{2}}+\left(\frac{F_{x f}}{k_{1}}\right)^{2}, \\
F_{y r}=k_{2}\left(\frac{v-b \omega}{u}\right) \sqrt{1-\left(\frac{F_{x r}}{\mu F_{z r}}\right)^{2}+\left(\frac{F_{x r}}{k_{2}}\right)^{2}},
\end{array}\right.
$$

where $k_{2}$ is the synthesized stiffness of rear tires, $\mu$ is the friction coefficient. 
Table 1. Parameter and definition

\begin{tabular}{|c|c|}
\hline Parameter & Definition \\
\hline$v$ & Lateral speed \\
\hline$u$ & Yaw rate of vehicle \\
\hline$\omega_{r}$ & Vehicle mass \\
\hline$m$ & Moment of inertia around $z$ axis \\
\hline$I_{z}$ & Moment of inertia of steering system \\
\hline$k_{w}$ & Distances of front axle from center of gravity \\
\hline$I_{w}$ & Distances of rear axle from center of gravity \\
\hline$a$ & Synthesized stiffness of rear tire \\
\hline$b$ & Steering system transmission ratio \\
\hline$k_{1}$ & Drag coefficient \\
\hline$k_{2}$ & Front wheel aligning arm of force \\
\hline$i$ & Front steering angle \\
\hline$c_{w}$ & Steering rate \\
\hline$\xi_{i}$ & Steering torque \\
\hline$\delta$ & The heading angle of the vehicle \\
\hline$p$ & \\
\hline$T_{s w}$ & \\
\hline$\theta$ & \\
\hline
\end{tabular}

Taking the effect of the longitudinal load transfer on the front and rear axles into account, the vertical forces of the front and rear wheels are:

$$
\left\{\begin{array}{l}
F_{z f}=\frac{m g b-\left(F_{x f}+F_{x r}\right) h_{g}}{a+b}, \\
F_{z r}=\frac{m g a+\left(F_{x f}+F_{x r}\right) h_{g}}{a+b},
\end{array}\right.
$$

where $h_{g}$ is the height of the center of gravity.

The vehicle velocity in the body coordinate is projected in the Earth coordinate defined by $x$ and $y$ as:

$$
\left\{\begin{array}{l}
\dot{x}=u \cos \theta-v \sin \theta \\
\dot{y}=v \cos \theta+u \sin \theta
\end{array}\right.
$$

According to Eq. (1) and Eq. (4), the state equation can be described as:

$\dot{x}=f[x(t), z(t)]$,

where $x(t)$ and $z(t)$ are the states and the input which are denoted respectively as $x(t)=[u(t), v(t), \omega(t), \delta(t), p(t), x(t), y(t), \theta(t)]^{T}, z(t)=\left[T_{s w}(t)\right]^{T}$.

\subsection{Constrains}

The constraints of initial and terminal states are:

$$
\begin{aligned}
& x\left(t_{0}\right)=\left[u_{0}, 0,0,0,0,0,0,0\right]^{T}, \\
& {\left[x_{2}\left(t_{f}\right), x_{3}\left(t_{f}\right), x_{4}\left(t_{f}\right), x_{5}\left(t_{f}\right), x_{8}\left(t_{f}\right)\right]^{T}=[0,0,0,0,0]^{T} .}
\end{aligned}
$$

In order to avoid pitch and yaw of the vehicle, the path constraint is [17]: 
$\frac{u^{2} \delta}{(a+b)\left(1+K u^{2}\right) g} \leq \frac{D}{2 h_{g}}$,

where $D$ is the wheel base, $K$ is the stability factor.

The constraints on $F_{x f}, F_{x r}$ are imposed when the vehicle is driven by the front wheels [18]:

$\left\{\begin{array}{l}F_{x f} \leq \frac{\mu m g b}{a+b+\mu h_{g}}, \\ F_{x r}=0 .\end{array}\right.$

When all the wheels are assumed as lock-braking, the constraints on $F_{x f}, F_{x r}$ can be rewritten in the following manner:

$\left\{\begin{array}{l}F_{x f} \geq-\frac{\mu m g\left(b+\mu h_{g}\right)}{a+b}, \\ F_{x r}=\frac{a-\mu h_{g}}{b+\mu h_{g}} F_{x f} .\end{array}\right.$

The boundary constraint is:

$z_{\min } \leq z \leq z_{\max }$

where $z_{\min }$ and $z_{\max }$ are the lower and upper limit values of the steering torque.

\subsection{Optimal control object of path tracking problem}

The minimum time problem of path tracking can be regarded as the optimal control problem in the control theory. The control target is to control the control variables to ensure the vehicle passing the given path within the minimum time. Therefore, the minimum time performance indicator is [19]:

$J=\int_{t_{0}}^{t_{f}} d t$

where $t_{0}$ and $t_{f}$ are the initial and final time.

\section{Adaptive Gaussian pseudospectral method}

\subsection{Continuous Bolza type problem}

The minimum time optimal control problem can be treated as a Bolza problem [16].

The Bolza cost function is:

$J=\psi\left(\mathbf{x}\left(t_{0}\right), t_{0}, \mathbf{x}\left(t_{f}\right), t_{f}\right)+\int_{t_{0}}^{t_{f}} g(\mathbf{x}(t), \mathbf{z}(t), t) d t$

where $t_{0}$ and $t_{f}$ are the initial and final time, $\mathbf{x}(t) \in \mathbf{R}^{n}, \mathbf{z}(t) \in \mathbf{R}^{m}$.

The dynamic and boundary constraints as well as inequality path constraint are:

$\dot{\mathbf{x}}=f(\mathbf{x}(t), \mathbf{z}(t), t), \quad t \in\left[t_{0} t_{f}\right]$,

$\varphi\left(\mathbf{x}\left(t_{0}\right), t_{0}, \mathbf{x}\left(t_{f}\right), t_{f}\right)=0$, 
$C[\mathbf{x}(t), \mathbf{z}(t), t] \leq 0, \quad t \in\left[t_{0} t_{f}\right]$

In Eqs. (13)-(16), the function $\psi, g, f, \varphi$ and $C$ are defined as follows:

$\psi: R^{n} \times R \times R^{n} \times R \rightarrow R$,

$g: R^{n} \times R^{m} \times R \rightarrow R$,

$f: R^{n} \times R^{m} \times R \rightarrow R^{n}$

$\varphi: R^{n} \times R \times R^{n} \rightarrow R \rightarrow R^{q}$,

$C: R^{n} \times R^{m} \times R \rightarrow R^{c}$.

The indirect method transforms the above Bolz problem into a Hamiltonian boundary problem by introducing the Hamiltonian function based on the Ponte Liya maximum principle. The Hamiltonian function is defined as:

$H(\mathbf{x}, \mathbf{z}, \boldsymbol{\lambda}, \boldsymbol{\mu})=g+\boldsymbol{\lambda}^{T} f-\boldsymbol{\mu}^{T} C$.

The optimal trajectories of the whole state variables and control variables can be obtained by solving a two-point boundary value problem composed of Hamiltonian equations, boundary conditions and path constraints.

\subsection{AGPM}

Gaussian pseudo-spectral method (GPM) separates the state variables and control variables on a series of collocations, and then uses the Lagrangian interpolation basis function to approximate the state variables and control variables with the discrete points as nodes. The derivative of the state variable to time can be approximated by deriving the global interpolation polynomial. The integral term in the performance index can be approximated by the Gaussian integral formula with high accuracy. The terminal state is obtained by integral of the initial state and the right function. After the above numerical approximation, the continuous optimal control problem can be transformed into a discrete nonlinear programming problem under a series of algebraic constraints.

GPM takes the K-order Legendre-Gauss point and the beginning and ending points as nodes to constitute $K+1$ Lagrangian interpolation polynomials. So the state and control variables can be approximated as [20]:

$\mathbf{x}(\tau)=\mathbf{X}(\tau)=\sum_{i=0}^{N} L_{i}(\tau) \mathbf{X}\left(\tau_{i}\right)$,
$\mathbf{z}(\tau)=\mathbf{Z}(\tau)=\sum_{i=0}^{N} L_{i}^{*}(\tau) \mathbf{Z}\left(\tau_{i}\right)$,

where $L_{i}(\tau)$ is Lagrange interpolating polynomials, $\mathbf{x}\left(\tau_{i}\right)=\mathbf{X}\left(\tau_{i}\right), \mathbf{z}\left(\tau_{i}\right)=\mathbf{Z}\left(\tau_{i}\right)$.

For the state variable approximation Eq. (18), the kinetic differential constraint equation can be transformed into the following algebraic constraint:

$\mathbf{D X}-\frac{t_{0}-t_{f}}{2} F\left(\mathbf{X}^{L G}, \mathbf{Z}^{L G}, \tau^{L G}, t_{0}, t_{f}\right)=0$,

where $\mathbf{D} \in \mathbf{R}^{n \times(n+1)}$.

The interpolation nodes in GPM include $N$ LG points, $\tau_{1}, \tau_{2} \cdots, \tau_{N}$, the initial point $\tau_{0} \equiv-1$, and the final point $\tau_{f} \equiv 1$. Since $X_{f}$ is absent in the state approximation, it must be constrained in other ways to ensure that it satisfies the state dynamic equation. This is accomplished by including an additional constraint that relates the final state to the initial state via a Gauss quadrature. According to the state dynamics: 
$\mathbf{x}\left(\tau_{f}\right)=\mathbf{x}\left(\tau_{0}\right)+\int_{-1}^{1} f(\mathbf{x}(\tau), \mathbf{z}(\tau), \tau) d \tau$

The integral function which is developed by Gauss-Lobatto integral approximation formula with the highest accuracy can be described as:

$\mathbf{X}_{N+1}=\mathbf{X}\left(\tau_{N+1}\right)=\mathbf{X}_{0}+\frac{t_{f}-t_{0}}{2} \omega_{k} F$,

where $\tau_{i}$ is the LG point, $\omega_{k}$ are the Gauss weights.

Then, the integral term in Eq. (13) can be approximated with a Gauss quadrature as before:

$J=\psi\left(\mathbf{X}_{0}, t_{0}, \mathbf{X}_{f}, t_{f}\right)+\frac{t_{f}-t_{0}}{2} \sum_{k=1}^{N} \omega_{k} g\left(\mathbf{X}_{k}, \mathbf{z}_{k}, \tau_{k}, t_{0}, t_{f}\right)$.

The boundary condition and the path constraint condition can be obtained at the interpolation points:

$\varphi\left(\mathbf{X}\left(\tau_{0}\right), t_{0}, \mathbf{X}\left(\tau_{f}\right), t_{f}\right)=0$,

$C\left[\mathbf{X}\left(\tau_{k}\right), \mathbf{Z}\left(\tau_{k}\right), \tau_{k}, t_{0}, t_{f}\right] \leq 0, \quad(k=1, \cdots, N)$.

Finally, the vehicle path tracking with the minimum time problem is defined as a NLP by the cost function of Eq. (23), the algebraic constraints of Eq. (20), Eq. (24), and Eq. (25).

GPM is a pseudospectral method based on the global interpolation polynomial. In order to improve the approximation accuracy, the above-mentioned global approximation pseudospectral method must increase the time node which will greatly increase the number of design variables and then will increase the calculation time. In the region where the approximation accuracy is smaller than the specified accuracy, the proposed pseudospectral method can improve the approximation accuracy by adding a fragment or a node in the region. However, the AGPM does not exist in the area where the accuracy satisfies the requirement, which contributes to improving computational efficiency.

Compared with the GPM mentioned in the previous section, the AGPM divides the whole problem into several fragments, and the GPM is applied in each segment. It is assumed that the problem contains $S$ fragments and $N$ collocation points:

$N=\sum_{i=1}^{s} N_{i}$

In this case, the kinetic approximation in Eq. (20) can be expressed as:

$$
\left[\begin{array}{llll}
D_{1} & 0 & \cdots & 0 \\
0 & D_{2} & \cdots & 0 \\
\vdots & & \ddots & \vdots \\
0 & \cdots & 0 & D_{s}
\end{array}\right] X=\frac{1}{2}\left[\begin{array}{llll}
t_{1}-t_{0} & 0 & \cdots & 0 \\
0 & t_{2}-t_{1} & \cdots & 0 \\
\vdots & & \ddots & \vdots \\
0 & \cdots & 0 & t_{s}-t_{s-1}
\end{array}\right] F
$$

It is obvious that the differential matrix of the segmentation collocation method (Eq. (27)) contains relatively few nonzero elements and is sparser than the differential matrix in the global configuration method of Eq. (20) contributing to improve the computation efficiency of the nonlinear programming algorithm and to reduce the calculation time. However, the reduction of the configuration point may reduce the corresponding approximation accuracy within each segment. On the other hand, the increase of the configuration point within each segment will make 
the whole nonlinear programming problem become more complicated and increase the computation time. In order to minimize the calculation time under the premise of ensuring the approximation accuracy, the AGPM automatically increases the configuration point or the fragment in the region where the accuracy does not meet the requirements. However, the AGPM does not change in the area where the accuracy satisfies the requirement to ensure the approximation accuracy while reducing the calculated pressure. In the region where the approximation accuracy does not meet the requirements, the AGPM determines whether the segment or the configuration point should be added by judging the form of the midpoint residual matrix. Firstly, the midpoint time $\bar{t}_{i}$ of each segment can be expressed as:

$\bar{t}_{i}=\frac{t_{i}+t_{i+1}}{2}, \quad\left(i=1, \cdots, N_{s}-1\right)$.

It is set that $\overline{\mathbf{X}} \in \mathbf{R}^{\left(N_{S}-1\right) \times n}$ and $\overline{\mathbf{Z}} \in \mathbf{R}^{\left(N_{S}-1\right) \times m}$ are state and control variable matrixes respectively at the time of $\bar{t}_{i}$. The midpoint residual matrix $\mathbf{R}$ is defined as:

$\mathbf{R}=\left|\overline{\mathbf{D}} \overline{\mathbf{X}}-\frac{t_{s}-t_{s-1}}{2} \mathbf{F}\left(\overline{\mathbf{X}}, \overline{\mathbf{Z}}, \bar{\tau}, t_{s}, t_{s-1}\right)\right| \in \mathbf{R}^{\left(N_{s}-1\right) \times n}$,

where $\overline{\mathbf{D}} \in \mathbf{R}^{\left(N_{S}-1\right) \times\left(N_{S}-1\right)}$ is the differential matrix at $\overline{\mathbf{\tau}}=\left(\bar{\tau}_{1}, \cdots, \bar{\tau}_{N_{S}-1}\right)$.

The midpoint residual $\beta$ is defined as:

$\boldsymbol{\beta}=\left[\beta\left(\bar{t}_{1}\right), \cdots, \beta\left(\bar{t}_{N_{s}-1}\right)\right]=\frac{\mathbf{r}}{\bar{r}^{\prime}}$

where column vector $\mathbf{r} \in \mathbf{R}^{\left(N_{S}-1\right) \times 1}$ is a column matrix containing the largest residual term in the midpoint residual matrix $\mathbf{R} ; \bar{r}$ is the mean arithmetic value of $\mathbf{r}$, that is $\bar{r}=\frac{\sum_{i=1}^{N_{S}-1} r\left(t_{i}\right)}{N_{S}-1}$. When each element of the midpoint residual vector $\boldsymbol{\beta}$ is equivalent, it is called the uniform residual vector; otherwise, it is called the non-uniform residual vector.

Let $\varepsilon$ be the specified allowable deviation, and take into account the case where the maximum residual in Eq. (30) is greater than $\varepsilon$. When the element's positions in the consistent residual vector $\boldsymbol{\beta}$ exceed the specified threshold $\rho(\rho>1)$, the placement point increase method within the segment is used to improve the approximation accuracy. That is $N_{s}^{\prime}=N_{s}+S$, where $S$ is the number of added nodes.

When the element's positions in the non-uniform residual vector $\boldsymbol{\beta}$ exceed the maximum allowable error $\rho$, the segment addition method at the time corresponding to the maximum element of the residual vector $\boldsymbol{\beta}$ is proposed to improve the approximation accuracy. If there are multiple largest elements, it is necessary to add the fragment at the corresponding moment of each largest element to improve the approximation accuracy.

The AGPM automatically adds a configuration point or fragment by automatically determining the value and form of the residual vector $\boldsymbol{\beta}$ until the accuracy satisfies the requirement in areas where the accuracy does not meet the requirements. In particular, the AGPM can ensure better performance in improving the approximation accuracy and the operation speed of the path tracking optimization problem.

The control strategy of the AGPM is:

Step 1: Initializing parameters and setting initial number of the collocations.

Step 2: The optimal control problem at the set collocations is discretized and transformed to a NLP problem.

Step 3: Solving the NLP problem.

Step 4: Determining if the dynamic constraint, the path constraint, the state and the control constraint at the midpoint of each element satisfy the given allowable deviation $\varepsilon$. If it is satisfied, 
the iteration stops. If not, step 5 is proceed.

Step 5: Determining if each element in the normalized midpoint residual vector $\boldsymbol{\beta}$ is smaller than the midpoint residual index $\rho$. If it is satisfied, step 6 is proceed. If not Step 7 is proceed.

Step 6: Increasing the number of collocations in the unit. That is to say, increasing the order of the Legendre interpolation polynomial, and then going to Step 3.

Step 7: Refining the corresponding unit to multiple units and going to Step 3.

The control block diagram is shown in Fig. 2.

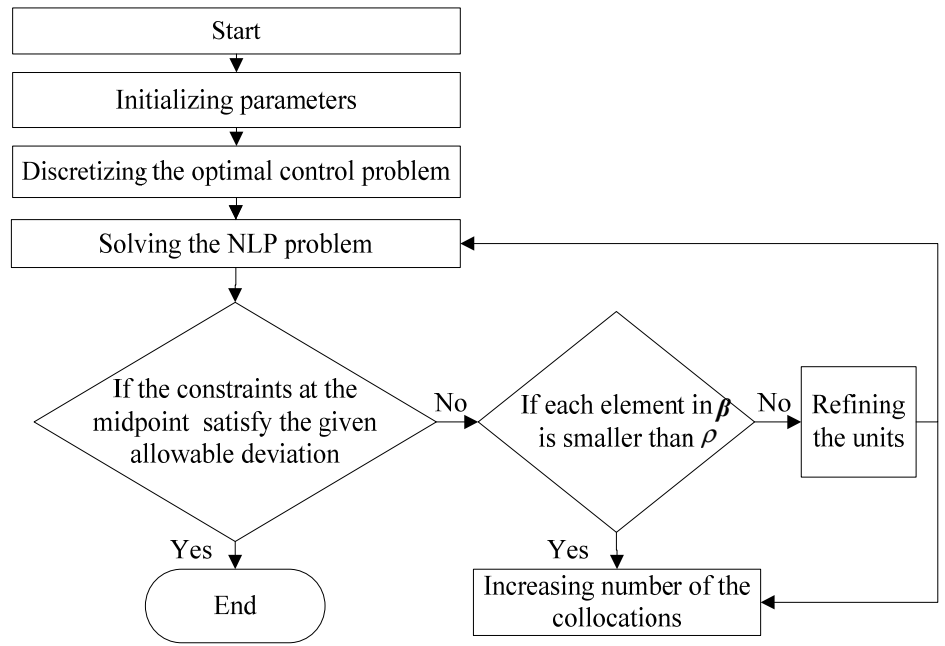

Fig. 2. The control block diagram of the AGPM

\section{Numerical simulation and experimental verification}

\subsection{Simulation result}

Simulation is carried out in Matlab software to verify the method.

The parameters of the real vehicle used in the simulation are shown in Table 2.

Table 2. Simulation parameters

\begin{tabular}{|c|c|c|}
\hline Symbol & Value & Unit \\
\hline$m$ & 1500 & $\mathrm{~kg}$ \\
\hline$I_{z}$ & 2080 & $\mathrm{~kg} \cdot \mathrm{m}^{2}$ \\
\hline$a$ & 1.185 & $\mathrm{~m}$ \\
\hline$b$ & 1.283 & $\mathrm{~m}$ \\
\hline$i$ & 20 & \\
\hline$C_{\varphi 1}$ & 63237 & $\mathrm{~N} \cdot \mathrm{m} / \mathrm{rad}$ \\
\hline$C_{\varphi 2}$ & 31458 & $\mathrm{~N} \cdot \mathrm{m} / \mathrm{rad}$ \\
\hline$c_{f}$ & -60533 & $\mathrm{~N} / \mathrm{rad}$ \\
\hline$c_{r}$ & -110185 & $\mathrm{~N} / \mathrm{rad}$ \\
\hline$h_{g}$ & 0.53 & $\mathrm{~m}$ \\
\hline
\end{tabular}

The tracked path is prescribed as the pylon course slalom test road shown in Fig. 3. A vehicle travels along the pylon course slalom test road. The vehicle should pass through the prescribed road within the minimum time.

In realistic driving process, the ideal target drivers' trajectory should be described as a curve of order three with continuous first-order derivative: 
$f(t)= \begin{cases}0, & t \in t_{1} \\ B(1.0+\sin \omega t) / 2.0, & t \in t_{2}, \\ B \cos \omega t, & t \in t_{3} \\ B(-1.0+\sin \omega t) / 2.0, & t \in t_{4} \\ 0, & t \in t_{5}\end{cases}$

From Eq. (31) it is easy to obtain the relationship between $y$, i.e. $f(x)$ and $x$ by substituting $t$ with $x / u$.

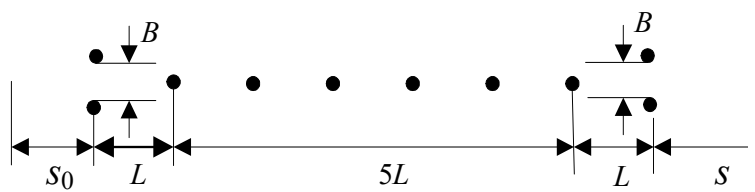

Fig. 3. Pylon course slalom test road (• stands for stake), where $s_{0}=L=2 u, s=3 u, u$ is the longitudinal speed. $B$ is the rotary distance, $B=2.46 \mathrm{~m}$. $L$ is the distance between the stakes

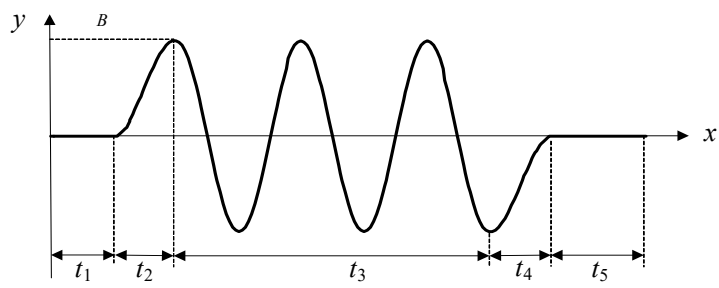

Fig. 4. Fitted pylon course slalom test road

The optimization is calculated by SQP algorithm and MATLAB software in a computer which CPU is $2.8 \mathrm{GHz} /$ Pentium IV, and operating system is Window XP. The minimum time for the vehicle accomplishing the pylon course slalom test road process is 14.9 seconds after 15 iterations.

Fig. 5 describes the result of the lateral distance of traveling along the pylon course slalom test road. It can be seen from the figure that the vehicle can successfully complete the pylon course slalom test road process without crossing the road boundary conditions with the speed of $90 \mathrm{~km} / \mathrm{h}$ indicating good control performance of the proposed method.

Fig. 6 is the simulation result of steering torque. It can be seen from the figure that there are peak values at $140 \mathrm{~m}, 175 \mathrm{~m}, 240 \mathrm{~m}$, and $280 \mathrm{~m}$, indicating that the driver manipulates the car strenuously.

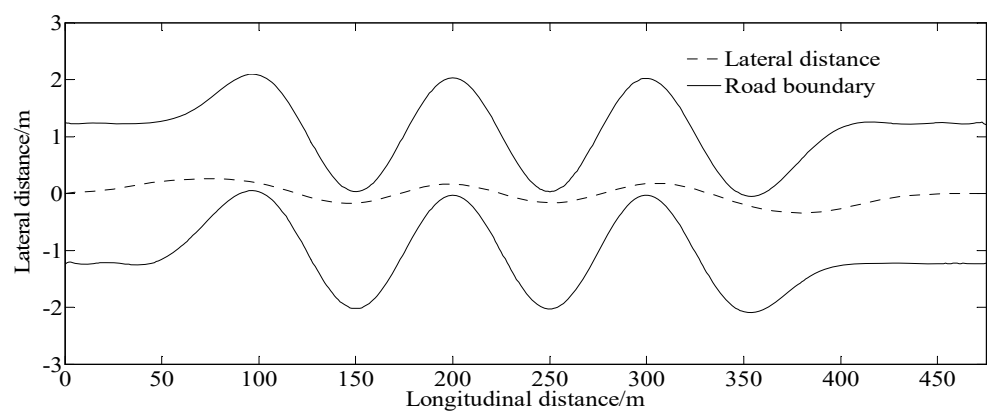

Fig. 5. Lateral distance

Fig. 7 is the simulation result of the traction force of front wheel. The figure indicates that the traction force of front wheel keeps growing until $1.63 \mathrm{kN}$. However, it can be seen from the curve that the curve slope keeps being small until 0 at $400 \mathrm{~m}$.

Fig. 8 is the simulation result of the longitudinal velocity. It is shown that longitudinal velocity 
keeps growing until $127 \mathrm{~km} / \mathrm{h}$. So, the driver should be cautious about maneuvering the car.

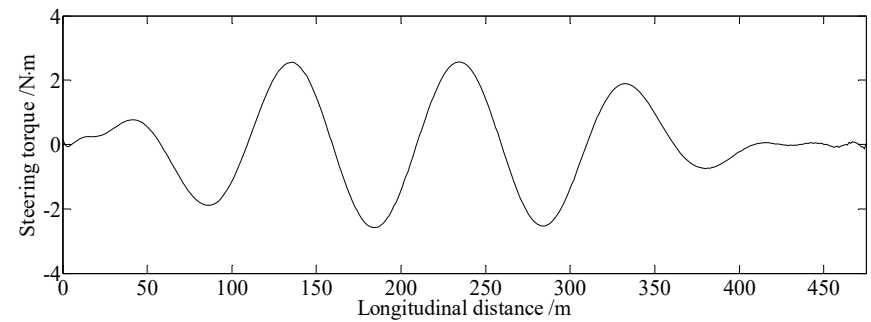

Fig. 6. Steering torque

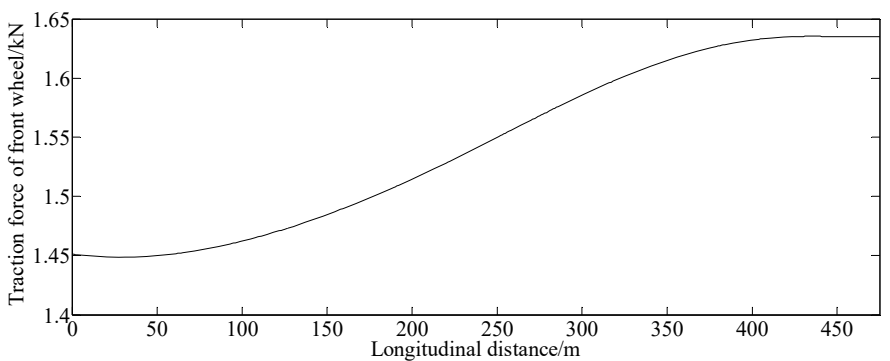

Fig. 7. Traction force of front wheel

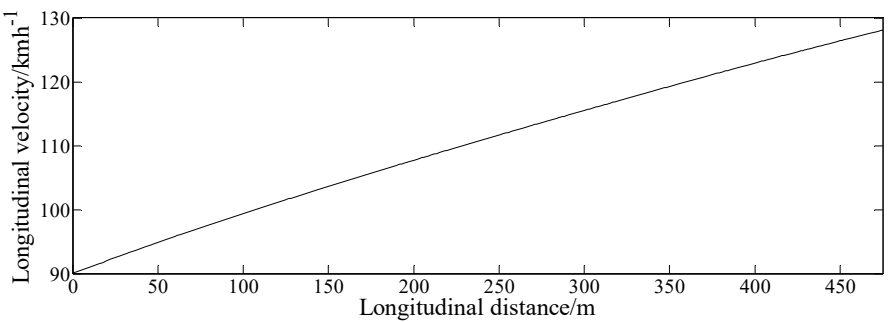

Fig. 8. Longitudinal velocity

\subsection{Evaluation of obstacle avoidance capability}

In order to compare the obstacle avoidance capability of different vehicles in the same path trajectory conditions, another vehicle which is remarked as vehicle 2 (The vehicle referred above is remarked as vehicle 1) completes the pylon course slalom test road process without crossing the road boundary conditions with the speed of $90 \mathrm{~km} / \mathrm{h}$. And the parameters of vehicle 2 used in the simulation are shown in Table 3.

Table 3. Simulation parameters

\begin{tabular}{|c|c|c|}
\hline Symbol & Value & Unit \\
\hline$m$ & 1265 & $\mathrm{~kg}$ \\
\hline$I_{z}$ & 1800 & $\mathrm{~kg} \cdot \mathrm{m}^{2}$ \\
\hline$a$ & 1.170 & $\mathrm{~m}$ \\
\hline$b$ & 1.195 & $\mathrm{~m}$ \\
\hline$i$ & 20 & \\
\hline$C_{\varphi 1}$ & 60544 & $\mathrm{~N} \cdot \mathrm{m} / \mathrm{rad}$ \\
\hline$C_{\varphi 2}$ & 32750 & $\mathrm{~N} \cdot \mathrm{m} / \mathrm{rad}$ \\
\hline$c_{f}$ & -60042 & $\mathrm{~N} / \mathrm{rad}$ \\
\hline$c_{r}$ & -109295 & $\mathrm{~N} / \mathrm{rad}$ \\
\hline$h_{g}$ & 0.53 & $\mathrm{~m}$ \\
\hline
\end{tabular}


Fig. 9(a) is the simulation result of lateral displacement of the two vehicles. It can be seen that the lateral displacement of the two vehicles is almost coincident. The pattern of motion for the vehicles is that the vehicles travel along a straight line almost between the road borders. Fig. 9(b) is the simulation result of the steering torque of the two vehicles. It can also be seen from the figure that the amplitude of steering torque of vehicle 1 is larger than that of vehicle 2 . The obstacle avoidance capability can be evaluated objectively by calculating the minimum time of the vehicles completing the pylon course slalom test road process. The evaluation of obstacle avoidance capability is shown in Table 4.

Table 4. Evaluation of obstacle avoidance capability

\begin{tabular}{|l|c|c|c|}
\hline \multicolumn{2}{|c|}{ Comment contents } & \multicolumn{2}{c|}{ Vehicle } \\
\cline { 2 - 4 } & Vehicle 1 & Vehicle 2 \\
\hline Minimum time (s) & $90 \mathrm{~km} / \mathrm{h}$ & 14.9 & 15.6 \\
\hline Obstacle avoidance capability & Better & Poor \\
\hline
\end{tabular}

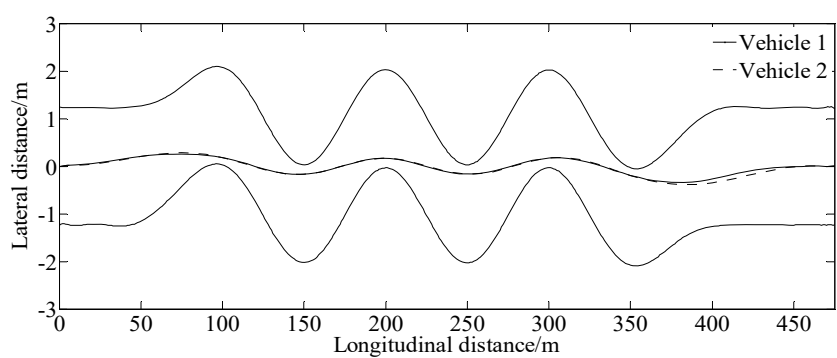

a) Lateral distance

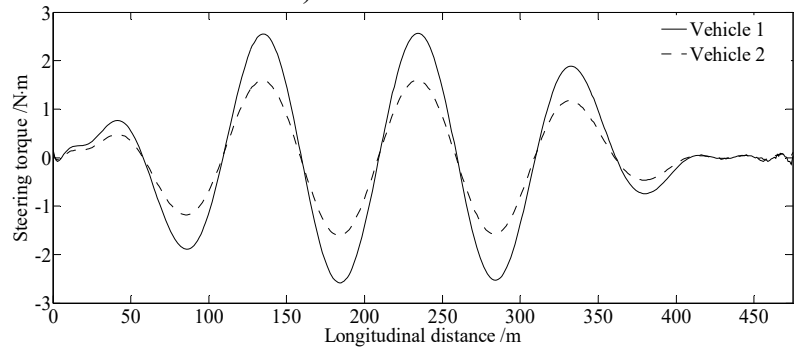

b) Steering torque

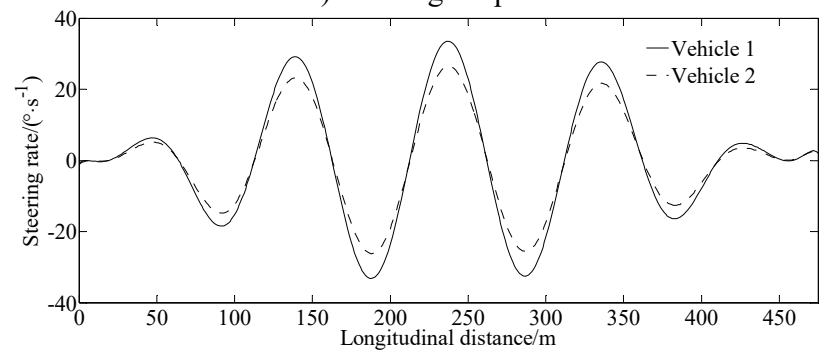

c) Steering rate

Fig. 9. Evaluation of obstacle avoidance capability

\subsection{Analysis of control efficiency}

In order to analyze the control efficiency of the AGPM compared with the traditional method (Direct parallel method [21] and LQR [22]), simulation with same conditions is established. The tracked trajectory is the double lane change test road.

The double lane change test road is described in Fig. 10. 


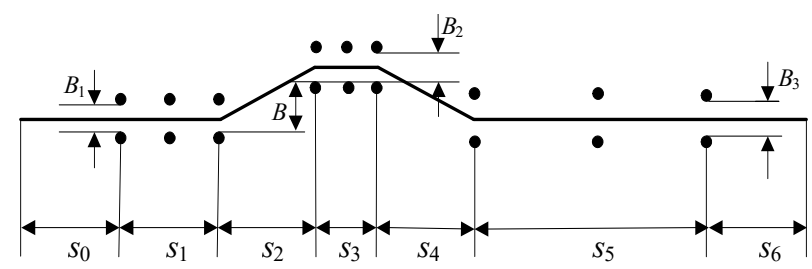

Fig. 10. Double lane change test road (• stands for stake), where $s_{0}=s_{1}=s_{2}=s_{4}=2 u, s_{3}=u$, $s_{5}=5 u, s_{6}=3 u . B$ is the distance of lane-change, $B=3.5 \mathrm{~m} . B_{1}, B_{2}$ and $B_{3}$ are the distances between the stakes, $B_{1}=1.1 H+0.25=2.12 \mathrm{~m}, B_{2}=1.2 H+0.25=2.29 \mathrm{~m}$, $B_{3}=1.3 H+0.25=2.46 \mathrm{~m}$, where $H$ is the width of the vehicle, $H=1.7 \mathrm{~m}$

In a realistic driving process, drivers' ideal target trajectory should be a low-level continuous smooth curve shown in Fig. 3. And also, according to ISO/TR3888-2004, the trajectory of the vehicle traveling along the pylon course slalom test road should be described as a curve of order three with continuous first-order derivative transformed with cubic splines fitting:

$f(x)= \begin{cases}0, & x \in s_{1}, \\ e_{0}+e_{1} x+e_{2} x^{2}+e_{3} x^{3}, & x \in s_{2}, \\ B, & x \in s_{3}, \\ e^{\prime}{ }_{0}+e^{\prime}{ }_{1} x+e^{\prime}{ }_{2} x^{2}+e^{\prime}{ }_{3} x^{3}, & x \in s_{4}, \\ 0, & x \in s_{5},\end{cases}$

where:

$a_{0}=s_{1}, \quad a_{1}=s_{1}+s_{2}, \quad a_{2}=s_{1}+s_{2}+s_{3}, \quad a_{3}=s_{1}+s_{2}+s_{3}+s_{4}$, $d=a_{1}-a_{0}, \quad d^{\prime}=a_{2}-a_{3}$,

$e_{0}=a_{0}^{2}\left(3 a_{1}-a_{0}\right) \frac{B}{d^{3}}, \quad e_{1}=-6 a_{0} a_{1} \frac{B}{d^{3}}, \quad e_{2}=3\left(a_{0}+a_{1}\right) \frac{B}{d^{3}}, \quad e_{3}=-2 \frac{B}{d^{3}}$,

$e_{0}^{\prime}=a_{3}^{2}\left(3 a_{2}-a_{3}\right) \frac{B}{d^{\prime 3}}, \quad e_{1}^{\prime}=-6 a_{3} a_{2} \frac{B}{d^{\prime 3}}, \quad e_{2}^{\prime}=3\left(a_{3}+a_{2}\right) \frac{B}{d^{\prime 3}}, \quad e_{3}^{\prime}=-2 \frac{B}{d^{\prime 3}}$.

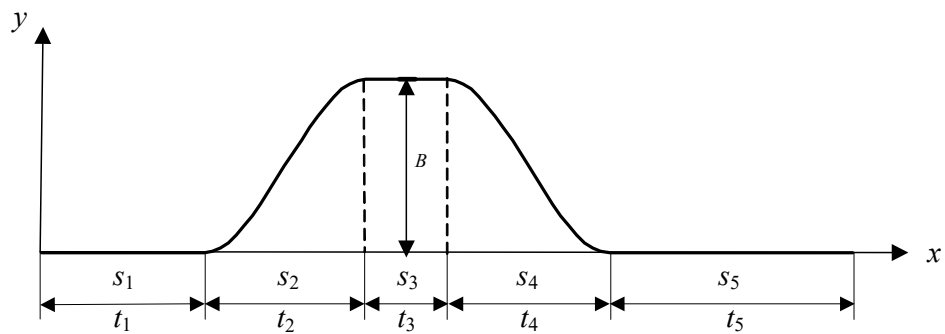

Fig. 11. Fitted double lane change test road

From Eq. (32) it is easy to obtain the relationship between $y$, i.e. $f(x)$ and $x$ by substituting $t$ with $x / u$ :

$f(t)= \begin{cases}0, & t \in t_{1}, \\ g_{0}+g_{1} t+g_{2} t^{2}+g_{3} t^{3}, & t \in t_{2}, \\ b, & t \in t_{3}, \\ h_{0}+h_{1} t+h_{2} t^{2}+h_{3} t^{3}, & t \in t_{4}, \\ 0, & t \in t_{5},\end{cases}$

where $g_{0}=e_{0} ; g_{i}=e_{i} u^{j}(j=1,2,3) ; h_{0}=e_{0}^{\prime} ; h_{i}=e_{i}^{\prime} u^{j}(j=1,2,3)$.

Simulation of lateral distance throughout the process of tracking the double lane change test 
road is shown in Fig. 12.

Fig. 12 indicates that the vehicle can track the double lane change test road well with speed of $105 \mathrm{~km} / \mathrm{h}$.

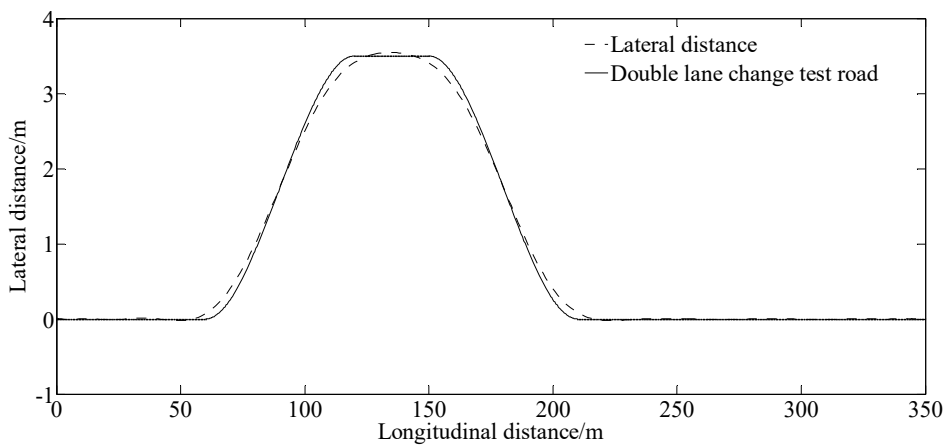

Fig. 12. Lateral distance of tracking the double lane change test road

The comparison results verifying the control efficiency of the proposed method is given in Fig. 13, which including the absolute error of the lateral distance between the simulation result and the double lane change test road as well as the Steering wheel angle.

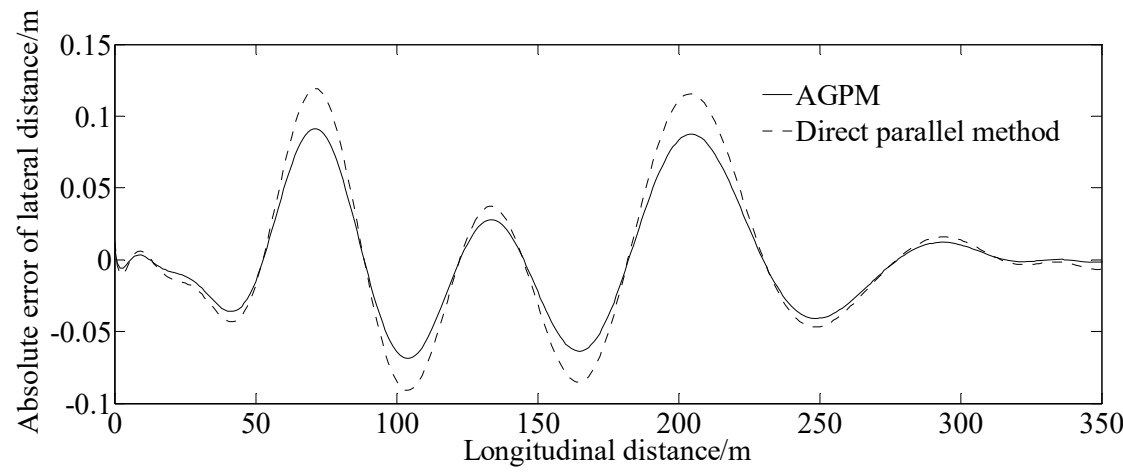

a) Absolute error of lateral distance

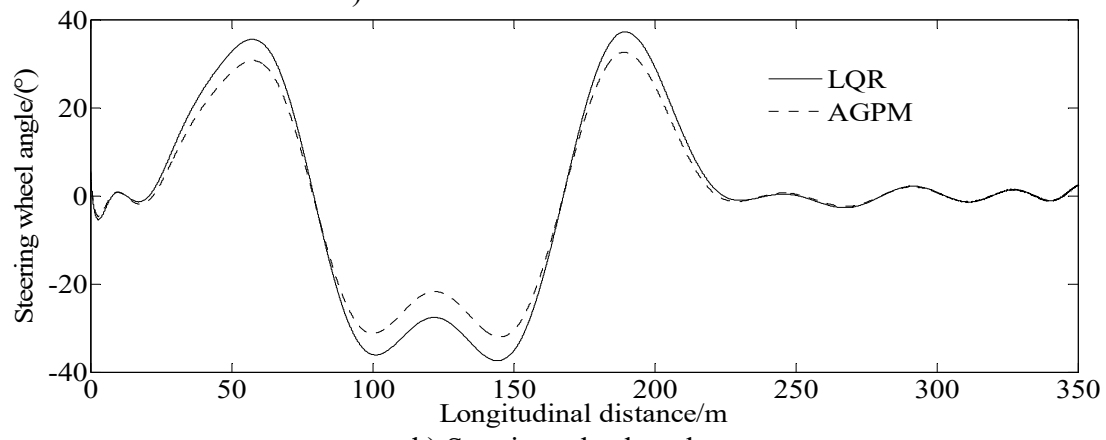

b) Steering wheel angle

Fig. 13. Evaluation of the control efficiency

From the absolute error of lateral distance curve in Fig. 13(a), it can be concluded that the maximum value of the error of lateral distance based on AGPM is less than that of the direct parallel method indicating that the AGPM is superior in calculation accuracy to the direct parallel method for solving the optimum path tracking problem of vehicle handling dynamics.

From Fig. 13(b), it can be concluded that the maximum value of the front steering angle based 
on the AGPM is less than that of the LQR controller. A more clear description of the evaluation of calculation accuracy is shown in Table 5.

Table 5. Evaluation of calculation accuracy

\begin{tabular}{|c|c|c|}
\hline \multirow{2}{*}{ Calculation results } & \multicolumn{2}{|c|}{ Maximum value (absolute value) } \\
\cline { 2 - 3 } & AGPM & LQR \\
\hline Steering wheel angle $\left(^{\circ}\right)$ & 30 & 36 \\
\hline
\end{tabular}

So, it is proved that there is higher control effect for the AGPM to solve the path tracking problem comparing with other traditional methods.

\subsection{Robustness verification in external disturbance environment}

In this paper, a C-Class Hatchback model in Carsim is used to implement the robustness verification of the proposed algorithm.

The longitudinal vehicle velocity, adhesion coefficient and maximum wind speed are set as $30 \mathrm{~m} / \mathrm{s}, 0.8$, and $50 \mathrm{~km} / \mathrm{h}$ respectively. The step gust is shown in Fig. 14.

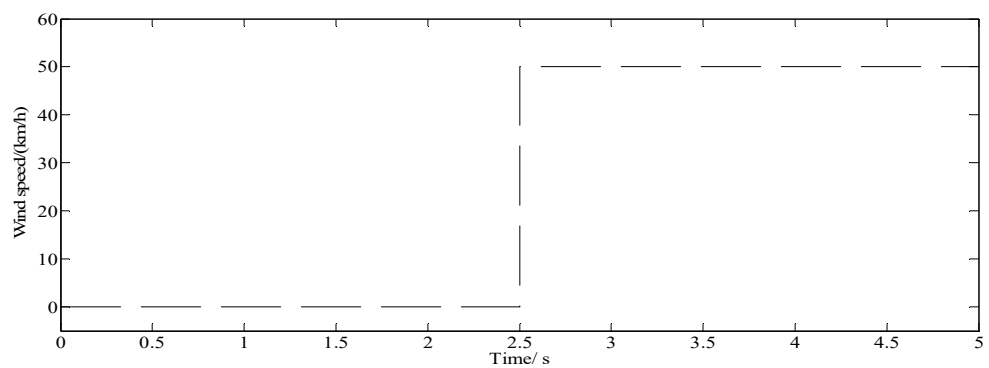

Fig. 14. Step gust

Fig. 15 shows that in the case of lateral wind, the vehicle still tracks the path well, indicating that the proposed algorithm can compensate robustness requirements in the external disturbance environment.

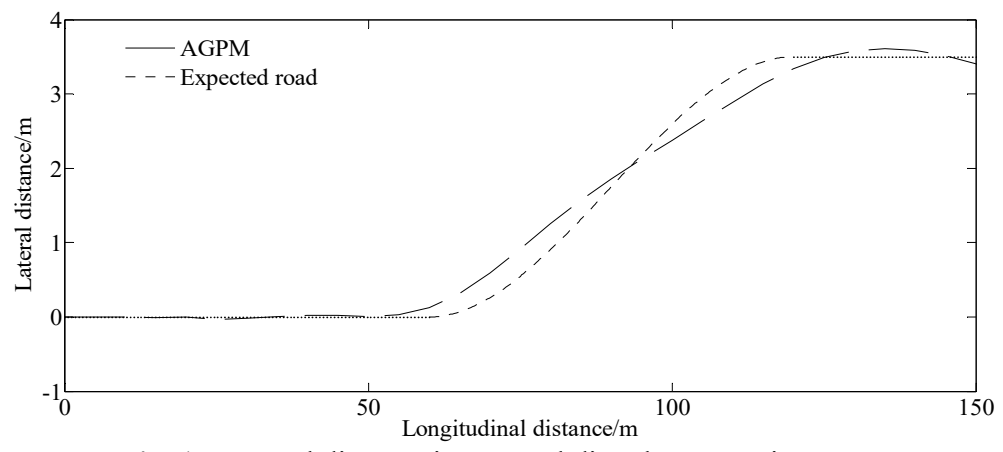

Fig. 15. Lateral distance in external disturbance environment

\subsection{Experimental result}

A real vehicle test was made to verify the correctness of the proposed algorithm. The block diagram of the test system and main measurement equipment is shown in Figs. 16-18. 


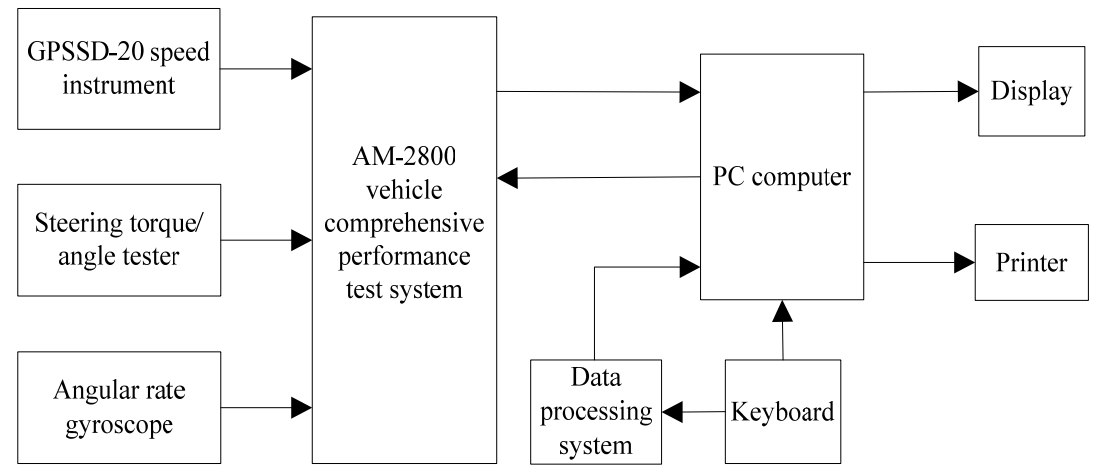

Fig. 16. Block diagram of test system

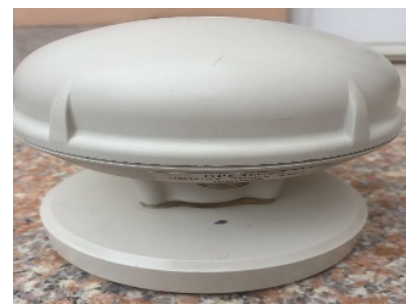

a) GPSSD-20 speed instrument

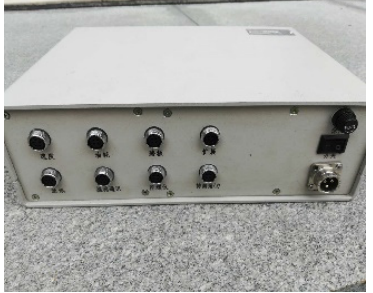

c) Comprehensive performance test system of AM-2800 vehicle

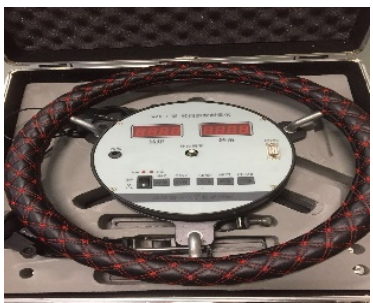

b) Steering torque/angle tester

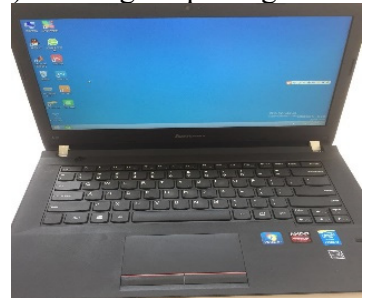

d) Lenovo computer

Fig. 17. Measurement equipment
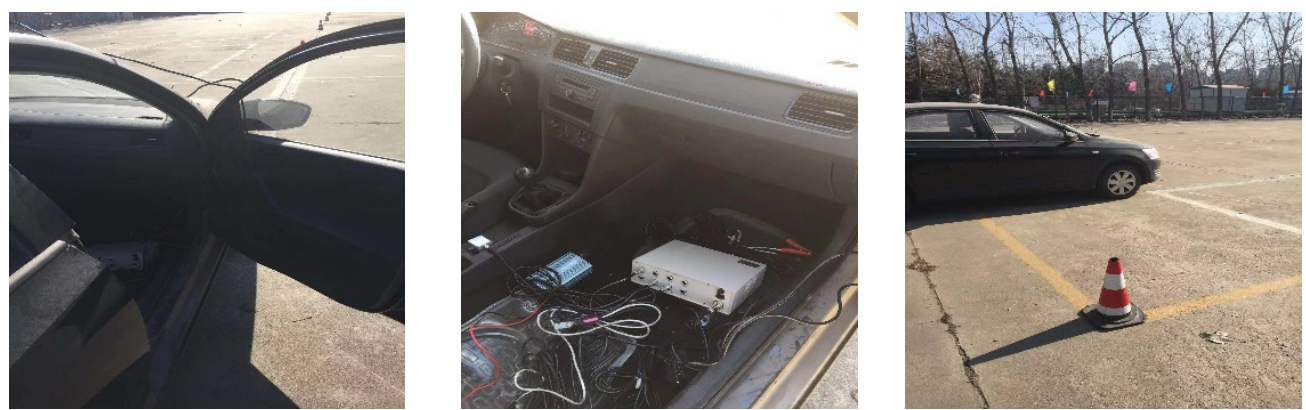

Fig. 18. Real test vehicle

The test procedure in accordance with ISO/TR3888-2004 is as follows:

Step 1: Arranging stakes shown in Fig. 3.

Step 2: Placing the related equipment shown in Fig. 17.

Step 3: A water injector is installed at the center of the front axle to spray water toward the ground in order to record the real traveling trajectory.

Step 4: Repeating Step 3 process 6 times.

The test values are shown in Figs. 19(a)-19(c). 


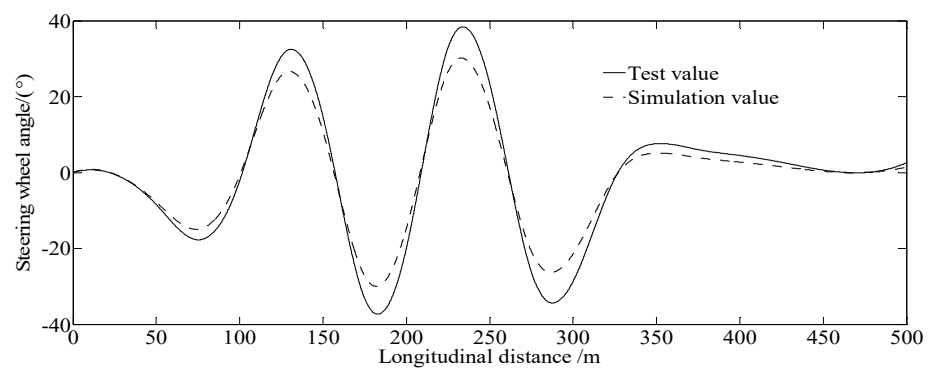

a) Steering wheel angle

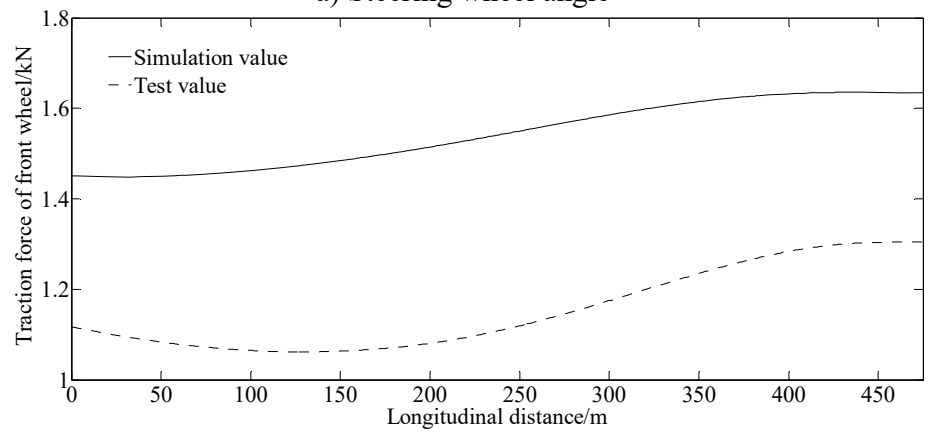

b) Traction force of front wheel

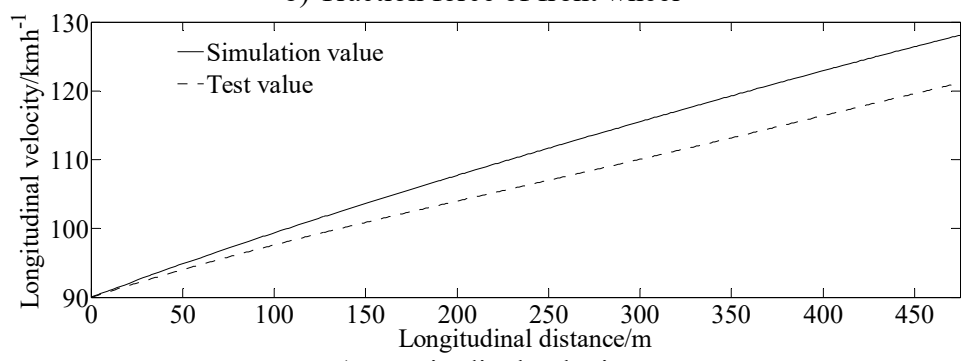

c) Longitudinal velocity

Fig. 19. Comparison of simulation and test value

It can be seen from Fig. 19(a) that both the test value and the simulation value of the steering wheel angle have peak values at $140 \mathrm{~m}, 175 \mathrm{~m}, 240 \mathrm{~m}$, and $280 \mathrm{~m}$, that indicates that the driver manipulates the car strenuously. When the driver manipulates the vehicle passing the pylon course slalom test road, the driver has to increase the steering wheel angle to avoid the stakes so the test value is bigger than that of the simulation. Fig. 19(b) shows that the test value and the simulation value increase as the longitudinal distance increase, this is because the driver must tread the throttle pedal to accelerate the vehicle to track the described trajectory within the minimum time. And the figure shows that the test value is smaller than the simulation value. This is because the driver must control the gears and throttle pedal to prevent the occurrence of side slip or rolling. At the same time, there are some errors in test equipment resulting in differences between test and simulation results. As shown in Fig. 19(c), both the test value and the simulation value of the longitudinal velocity increase as the longitudinal distance increases. This is because the driver must accelerate the vehicle to track the described path within the minimum time. But the test value is smaller than the simulation value. This is reasoned by that there are psychological problems in performing a certain impact on the test results for the driver driving the vehicle at a high speed. From Fig. 19(c), it is also can be seen that the error between the simulation and the real test values increases as the longitudinal distance increases. Both the simulation and the real test values tend to two lines almost with different slopes caused by driving skill and psychological burden under the condition of vehicle tracking the described path within the minimum time. At the same time, 
the two lines have the same starting coordinates $(0,25)$, that is why the error between the simulation and the real test values occurs for the longitudinal distance.

However, the simulation and the experimental values are consistent, verifying the correctness of the proposed algorithm.

The authors declare that there is no conflict of interests regarding the publication of this paper.

\section{Conclusions}

In this paper, the AGPM method is utilized to analyze the problem of vehicle tracking the desired path within the minimum time. Based on a 4-DOF simplified vehicle model, the AGPM method is proposed to solve the problem of path tracking with a maneuver as quickly as possible.

To verify the performance of the proposed method, a simulation is conducted, and its results show that the minimum time for the vehicle (vehicle 1) accomplishing the pylon course slalom test road process is 14.9 seconds after 15 iterations. And also, the proposed method can be used to research for an optimized trajectory directly. This is the main benefit of this method for path tracking control problem within the minimum time.

The results of comparison between the AGPM and other traditional methods (Direct parallel method and LQR) method show that there is higher control effect for the AGPM to solve the path tracking problem comparing with other traditional methods under the same computation conditions.

In future research, the aim would to be conduct comprehensive evaluation of driver's burden in the path tracking with minimum time problem and identify driver model parameters in the closed-loop method as well as verifying driver physiological limit. In the future, the proposed algorithm can be used to improve vehicle handling performance and provides valuable insight into the lane changes design work and can develop target tracking as well as collision avoidance techniques in the area of vehicle safety.

\section{Data availability}

The experimental data used to support the findings of this study are currently under embargo while the research findings are commercialized. Requests for data, [6/12 months] after publication of this article, will be considered by the corresponding author.

\section{Acknowledgement}

This paper was supported by the Science and Technology Program Foundation of Weifang under Grant 2015GX007. The first author gratefully acknowledges the support agency.

\section{References}

[1] Amer N. H., Hudha K., Zamzuri H. Adaptive modified Stanley controller with fuzzy supervisory system for trajectory tracking of an autonomous armoured vehicle. Robotics and Autonomous Systems, Vol. 105, Issue 1, 2018, p. 94-111.

[2] Mengyin F., Kai Z., et al. Collision-free and kinematically feasible path planning along a reference path for autonomous vehicle. IEEE Intelligent Vehicles Symposium (IV), Korea, 2015.

[3] Zambom A. Z., Collazos J. A. A., Dias R. Constrained optimization with stochastic feasibility regions applied to vehicle path planning. Journal of Global Optimization, Vol. 64, Issue 4, 2016, p. 803-823.

[4] Vilela D., Barbosa R. S. Numerical optimization methods applied to the concurrent problem of vehicle ride and handling. International Journal of Vehicle Systems Modelling and Testing, Vol. 8, Issue 4, 2013, p. 316-334.

[5] Zhang He, Yang S. W. Smooth path and velocity planning under 3D path constraints for car-like vehicles. Robotics and Autonomous Systems, Vol. 107, Issue 5, 2018, p. 87-99. 
[6] Yang I. B., Na S. G., Heo H. Intelligent algorithm based on support vector data description for automotive collision avoidance system. International Journal of Automotive Technology, Vol. 18, Issue 1, 2017, p. 69-77.

[7] Fu M. M., Ni J., Li X. Y., Hu J. B. Path tracking for autonomous race car based on G-G diagram. International Journal of Automotive Technology, Vol. 19, Issue 4, 2018, p. 659-668.

[8] Krid M., Benamar F., Lenain R. New explicit dynamic path tracking controller using generalized predictive control. International Journal of Control, Automation and Systems, Vol. 15, Issue 1, 2017 , p. 303-314.

[9] Amer N. H., Zamzuri Hairi, Hudha Khisbullah, et al. Path tracking controller of an autonomous armoured vehicle using modified Stanley controller optimized with particle swarm optimization. Journal of the Brazilian Society of Mechanical Sciences and Engineering, Vol. 40, Issue 104, 2018, p. 103-119.

[10] Guo J. H., Luo Y. G., Li K. Q., Dai Y. F. Coordinated path-following and direct yaw-moment control of autonomous electric vehicles with sideslip angle estimation. Mechanical Systems and Signal Processing, Vol. 105, Issue 1, 2018, p. 183-199.

[11] Memon K. R., Memon S., Memon B., et al. Real time implementation of path planning algorithm with obstacle avoidance for autonomous vehicle. 3rd International Conference on Computing for Sustainable Global Development, 2016, p. 2048-2053.

[12] Setiawan Y. D., Nguyen T. H., Pratama P. S., et al. Path tracking controller design of four wheel independent steering automatic guided vehicle. International Journal of Control, Automation and Systems, Vol. 14, Issue 6, 2016, p. 1550-1560.

[13] Zou Ting, Angeles J., Hassani Ferri Dynamic modeling and trajectory tracking control of unmanned tracked vehicles. Robotics and Autonomous Systems, Vol. 110, Issue 4, 2018, p. 102-111.

[14] Yu M., Xin G., Yu W., Qing G. LQR-GA controller for articulated dump truck path tracking system. Journal of Shanghai Jiaotong University (Science), Vol. 24, Issue 1, 2019, p. 78-85.

[15] Wang J. X., Wang J. M., Wang R. R., et al. Framework of vehicle trajectory replanning in lane exchanging with considerations of driver characteristics. IEEE Transactions on Vehicular Technology, Vol. 66, Issue 5, 2017, p. 3583-3596.

[16] Liu Y. J., Jiang J. S. Optimum path tracking control for inverse problem of vehicle handling dynamics. Journal of Mechanical Science and Technology, Vol. 30, Issue 8, 2016, p. 3433-3440.

[17] Zhang L. X., Zhao Y. Q., Song G. X. Research on inverse dynamics of vehicle minimum time maneuver problem. China Mechanical Engineering, Vol. 18, Issue 21, 2007, p. 2628-2632, (in Chinese).

[18] Jin Z. L., Weng J. S., Hu H. Y. Rollover stability of a vehicle during critical driving maneuvers. The Proceedings of the Institution of Mechanical Engineers, Part D: Journal of Automobile Engineering, Vol. 221, Issue 9, 2007, p. 1041-1049.

[19] Casanova D. On Minimum Time Manoeuvring: The Theoretical Optimal Lap. Cranfield, England, 2000.

[20] Sun Z. Y., Jin G., Zhang L., Yang X. B. SGCMG non-singularity trajectory programming algorithm based on adaptive gauss pseudospectral method. Journal of Astronautics, Vol. 33, Issue 5, 2012, p. 597-604.

[21] Vavrina M. A., Englander J. A., Ellison D. H. Global optimization of N-maneuver, high-thrust trajectories using direct multiple shooting. 26th AAS/AIAA Spaceflight Mechanics Meeting, United States, 2016.

[22] Chen, H. Study on path following control method for automatic parking system based on LQR. SAE International Journal of Passenger Cars - Electronic and Electrical Systems, Vol. 10, Issue 1, 2017, p. $41-49$. 